\title{
Niveles de interacción en la comunicación en Internet ${ }^{1}$
}

\author{
Diana M. Kiss de Alejandro \\ (Universidad de Los Lagos)
}

\section{Resumen}

Este trabajo presenta una categorización de los niveles de interacción que los usuarios de Internet establecen entre ellos mismos y con la tecnología que los convoca. Se plantea que aquellos flujos informativos y comunicacionales que circulan en Internet son variados y diversos y que están determinados no sólo por las características técnicas del equipamiento y los soportes materiales, sino, fundamentalmente, por las competencias de los usuarios, a través de las cuales se realiza más de un proceso de comunicación.

\begin{abstract}
This paper presents a chart of interaction levels on Internet users. These levels are established among themselves and the technology that summons the users. The investigation states that the communicational flows that circulate in Internet are varied, diverse and are determined by technologic characteristics of the equipment and material support but, fundamentally by the competition of the users. During these process, the users materialize more than one act of communication.
\end{abstract}

Palabras clave: Interacción, comunicación digital, Internet, comunicación educativa

Key words: Interaction, digital communication, Internet, educational communication

\section{INTERNET COMO OBJETO DE INVESTIGACIÓN EN AMÉRICA LATINA}

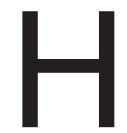

ace tan solo cuatro años la investigación en comunicación en América Latina apenas si consideraba las comunicaciones en Internet como objeto de estudio. En el año 2000 el Congreso de la Asociación Latinoamericana de Investigación de la Comunicación (ALAIC), celebrado en Santiago de Chile reunía a sólo media docena de trabajos que exploraron tímidamente los alcances de este "nuevo medio". Ese año quienes nos interesamos por las comunicaciones a través de "la red" participamos en los grupos de trabajo sobre Tecnologías de la Comunicación y Metodologías de la comunicación, pues en rigor en ninguno de los grupos en los cuales ALAIC venía trabajando contemplaban Internet como objeto de estudio de manera explícita. Las investigaciones presentadas se centraron, fundamentalmente, en los aspectos técnicos, económicos o políticos y el impacto en el nivel de las estructuras socioculturales, sobre todo los estudios sobre el uso y el crecimiento del sistema en los distintos países de América Latina.

\footnotetext{
${ }^{1}$ Trabajo realizado con el financiamiento de la Dirección de Investigación de la Universidad de Los Lagos. Concurso de Proyectos de Investigación 2002-2004.
} 
En el marco del evento académico en mención, uno de los trabajos que alertaba sobre la expansión de Internet como eje de investigación fue el presentado por Raúl Fuentes Navarro, quien presentó los resultados preliminares de un estudio respecto de los artículos sobre Internet en revistas académicas internacionales de ciencias sociales (consulta en ProQuest), cuyos datos pronosticaban que la investigación sobre Internet como medio de comunicación apenas comenzaba. Y así fue. En los últimos cinco años las investigaciones relativas a Internet en sus más diversas facetas, disciplinas y ejes de problematización se han reproducido de manera extraordinaria, a lo que se suma el interés de los sectores políticos, sociales, productivos, culturales y académicos, para incorporar las ventajas de la conectividad de un nuevo medio a sus respectivos programas y estrategias de desarrollo.

En el año 2002, en Santa Cruz de la Sierra, en Bolivia, ALAIC convocó finalmente a la presentación de trabajos en el Grupo "Internet y Sociedad de la Información" y desde entonces, los aportes han sido abundantes y variados, explorándose no sólo las elementos que determinan el consumo de los llamados "nuevos medios" o las TIC, sino explorando la reconstrucción teórica de la disciplina con la incursión del estudio de conceptos como la virtualidad, la interacción digital y la reconfiguración del contexto de recepción. De esta manera, pasamos del asombro inicial por la incursión de un nuevo medio, a la preocupación por la economía, por el conocimiento, por la política, la expansión del dominio tecnológico y por la dependencia económico-tecnológica, para preguntarnos por los usos, los modos de comunicarnos, el proceso de conocer, las reconstrucciones socioculturales, políticas, económicas o epistemológicas que implica una sociedad en red.

Hemos vuelto la mirada a las características, a las formas y modos de conocer, comunicar, de aprender y de aprehender, que provocan los medios digitales, a identificar los procesos paralelos, alternos y/o complementarios que emergen con el acceso y uso del "nuevo medio".

Para Wolton ${ }^{2}$ lo que aparece como comunicación en las redes son por lo menos cuatro cosas distintas:

· información-servicio

- información-ocio

- información-acontecimientos (noticias)

- información-conocimiento.

Si bien Wolton ${ }^{3}$ se refiere al contenido de los textos que se presentan en Internet y el uso que el destinatario hace de estos, su distinción nos lleva a preguntarnos sobre el significado mismo de la comunicación en la Red y si acaso no estamos frente a un espejismo, donde contemplamos como comunicación lo que no es.

2 WOLTON, D. "Internet, ¿y después?", Barcelona, Gedisa, 2000.

${ }^{3}$ Ibidem. 
La preocupación de este trabajo, que presenta los resultados de una investigación sobre los niveles de comunicación que se materializan a partir del uso de Internet por parte de estudiantes universitarios, es explorar de qué manera los usuarios se comunican diferenciadamente de acuerdo, tanto a los servicios que la red ofrece y de los cuales los usuarios se han apropiado, como a las propias competencias tecnológicas y las variables del contexto sociocultural en que los jóvenes se desenvuelven, variables que en definitiva determinan la naturaleza de la práctica comunicativa hoy en día.

\section{EL EJE DE LA INVESTIGACIÓN}

El trabajo es de naturaleza exploratoria ${ }^{4}$ y ha centrado su atención en la definición de los rangos de competencia que tienen los (las) estudiantes a partir de los niveles de escolaridad superior (año de ingreso a la Universidad), la carrera universitaria que cursan, los usos, funciones, frecuencia de uso y competencia tecnológica en la práctica de la comunicación a través de Internet, lo que nos ha llevado a identificar niveles de interacción desde la relación que el usuario establece con el soporte tecnológico-digital.

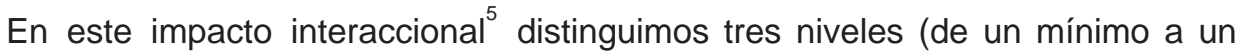
máximo de interacción) en la comunicación que establecen los usuarios con la Red, pues consideramos que en el universo de lo virtual, "lo que es no es lo que parece" ${ }^{\sharp,}$ y en el fenómeno de la interactividad en la relación usuario-tecnología, existen matices que es necesario precisar.

El objetivo de la investigación es identificar las características de los "niveles" de la comunicación interactiva a través de Internet, que en definitiva involucrarían, a su vez, los mecanismos de apropiación o procesos cognitivos imperantes en el usuario en su aproximación a los denominados "nuevos medios".

La recolección de datos se realizó con la aplicación de un cuestionario y entrevistas en profundidad donde se exploraron los elementos que determinarían el tipo de interacción que los usuarios establecen con Internet y las características de tales procesos. El universo estudiantil de la Universidad de Los Lagos, donde se desarrolló la investigación, es de 4.300 alumnos (as), el estudio se centró en los estudiantes de las ocho carreras pedagógicas del campus Osorno, con una población total de 1040 alumnos(as). Las entrevistas en profundidad se realizaron a una muestra no probabilística de dos estudiantes por carrera.

${ }^{4}$ DAHNKE, G. L. "Investigación y Comunicación " En Fernández-Collado, C. y Dahnke, G. L. (comps.) "La Comunicación Humana, ciencia social", México, McGraw-Hill, 1996, pp. 385-452.

${ }^{5}$ THOMPSON, J. "Ideología y cultura moderna. Teoría crítica social en la era de la comunicación de masas", México, UAM-Xochimilico, 1993.

${ }^{6}$ SANCHO, J. "Enfoques y funciones de las nuevas tecnologías para la información y la educación: lo que es no es lo que parece", En De Pablos, J. y Jiménez, F. "Nuevas tecnologías, comunicación audiovisual y educación", Barcelona, Cedenc, 1998, pp. 71-102. 


\section{INTERNET: ¿UN MEDIO DE COMUNICACIÓN O UN SISTEMA DE INFORMACIÓN?}

Internet nos plantea interrogantes infinitas, que van desde definir exactamente lo que es hasta explorar lo que se puede hacer. Nuestros cuestionamientos frente al sistema son recurrentes, aún no acabamos de definir si estamos frente a un nuevo medio, a un nuevo soporte para la comunicación, a un medio de comunicación, de información o sólo de entretenimiento. Elizabeth Saad sostiene que frente a Internet estamos ante un ambiente mutante, donde no se tienen paradigmas ni conceptos consolidados, más bien estamos delante de una "metadiscusión" donde todos, los que se involucran directa o indirectamente con el sistema se transforman en usuarios, adoptando el perfil de internauta, para satisfacer sus necesidades de contenido, interacción o diversos intercambios. Es decir, nos aproximamos el objeto de estudio desde nuestra directa experiencia con el sistema investigado.

En este medio "mutante" el umbral de la comunicación estaría dado, en un principio, por los rangos de participación del usuario en la construcción del mensaje o texto, o más bien en los niveles de interactividad materializados por el "internauta" en su navegación por la Red. El dilema entre proceso de comunicación y proceso de información nos lleva a explorar más allá de la respuesta y/o intervención del usuario (o del receptor en la nomenclatura tradicional) frente a un mensaje determinado para definir la "comunicabilidad" de un intercambio textual desde la perspectiva de otros elementos, como son los rangos de "control" sobre los contenidos y el proceso de apropiación del texto.

Esto es, las transformaciones estructurales que plantean las interacciones digitales nos conducen no sólo a denominar de forma distinta a los elementos del proceso de la comunicación, dado que estos multiplican las funciones dentro de la dinámica de exposición a los productos mediáticos. Ahora hablamos de procesos interactivos más que de procesos comunicativos, en nuestra referencia a la relación que establecemos con Internet.

Estamos frente a un cambio radical en las operaciones cognitivas y motoras que realiza el decodificador al relacionarse en forma individual y creativa con un canal de comunicación, que exige un compromiso mucho más explícito y directo de su parte, más allá de las operaciones sociales que implica la recepción activa de los mensajes presentados por los medios de comunicación colectiva tradicionales.

Por otra parte, no debemos olvidar que en el estudio de los procesos y las relaciones que convergen en la experiencia de la comunicación interpersonal mediatizada por herramientas tecnológicas digitales, "la lógica de los mensajes lineales se fragmenta para dar lugar a sistemas conceptuales basados en nociones como la multilinealidad, los nodos, nexos y redes", ${ }^{\prime \prime}$ con lo cual el simple intercam-

\footnotetext{
7 SAAD, E., Presença digital da universidade: em busca da excelência colectiva (2, mayo, 2005), Disponible en: http//njmt.incubadora.fapesp.br/portal/publi/beth/jornaluspdez00

${ }^{8}$ DE VECCHI, B. "Nuevas formas de vida, nuevas estructuras de comunicación", Hyperpage. Recuperado el 20 de diciembre del 2000, de http://www.kweb.it/hyperpage/biblio1.htm
} 
bio de datos se torna complejo y evidentemente un área de riesgo al momento de explicar lo que ocurre en el marco de las interacciones usuario-máquina, usuariotexto y usuario-usuario.

Respecto a la interactividad, Gutiérrez ${ }^{9}$ sostiene que las relaciones entre el usuario y la máquina pueden definirse como relaciones de control sobre los contenidos, donde la interactividad viene marcada por la capacidad de control que tiene el usuario y/o el sistema sobre el tipo de contenidos, su secuenciación, metodología, etc., identificando tres niveles de interactividad, por una parte aquellos donde es el sistema el que controla y otro donde la capacidad de decisión está en manos del usuario, y un tercer nivel donde el sistema es capaz de recoger y considerar las elecciones del usuario para la construcción de un nuevo texto.

La identificación de los niveles de interactividad que realiza el autor se orientan a analizar los procesos de participación de los usuarios en los sistemas de enseñanza multimedia. Sin embargo, en Internet una de las características es precisamente la interactividad $^{10}$, que junto a la desmasificación y a la asincronía dibujan un nuevo escenario para las comunicaciones con soporte tecnológico. La interactividad es entendida como la capacidad de los participantes en el proceso para intercambiar roles en la emisión de sus discursos, esto es, la interactividad se encuentra reducida a los servicios de comunicación sincrónica que la Red ofrece. Por su parte Kramer $^{11}$ sostiene que esta interactividad telemática presenta rasgos distintivos que deben ser considerados en la conceptualización del proceso comunicativo, como es que en Internet es posible interactuar con datos y no directamente con personas, es decir, se establecería una primera relación material más que personal. En este proceso se prescinde del aspecto ilocutivo o paralingüístico de la actividad simbólica, la interacción tiene el estatus de juego, el universo de datos legibles en la máquina es la exteriorización del principio de la intertextualidad, y se crea una nueva forma de memoria colectiva.

A partir de los resultados de la investigación hemos ampliado el espectro de los rangos de interacción presentes en la "navegación" del usuario por los servicios que ofrece Internet, proponiendo niveles de interactividad de acuerdo a las elecciones del internauta y las alternativas de intervención que la red ofrece actualmente.

\section{MODOS DE COMUNICARSE EN INTERNET}

En la interacción que los usuarios establecen con y a través de internet, identificamos distintos modos de comunicación, que agrupamos de la siguiente manera:

${ }^{9}$ GUTIÉRREZ, A. "Alfabetización digital. Algo más que ratones y teclas", Barcelona: Gedisa. 2003.

${ }^{10}$ WILLIAMS, F. y otros, "Research Methods and the New Media. New York: Free Press, 1998.

${ }^{11}$ KRAMER S. Dialogicita telemática? Tesi sula comunicazione en Internet, Rivista Sociología della Comunicazione, Istituto de Scienza dello Spettacole e Sociología della Comunicazione. Recuperado el 30 de octubre de 1997, de http://uiurb.it/ISCCS/dialogic.html 
1. De acuerdo a la inmediatez de la retroalimentación: Desde la perspectiva de la retroalimentación en línea los usuarios del sistema se comunican de forma tanto asincrónica como sincrónica. En ambas existe una interacción, cuya diferencia estará en los lapsos de tiempo que pasan entre la construcción del enunciado y la respuesta a éste, sin que intermedie en ello una alteración al proceso. La interacción se percibe como unidireccional por parte del usuario, quien es el que se comunica con "el otro":

"... me comunico por e-mail y chat";

“... utilizo el correo para comunicarme con otras personas...";

"... con el e-mail me comunico más rápido con mis compañeros...";

"... a veces lo utilizo, para la cosa el chat";

"... el correo ahí, el chat de repente";

"... escribo mails y uso el MSN".

2. De acuerdo a la iniciativa en la selección de datos: Los usuarios identifican la selección de datos en Internet como una forma de comunicación, pues son elegidos algunos textos de entre aquellos disponibles en la red y con ellos se construye implícitamente una respuesta ante un texto determinado. Es decir, los usuarios distinguen la selección de información como un modo de construir sus propios textos, completándose de esta manera el ciclo del proceso comunicativo, como es la articulación de una respuesta a un mensaje en circulación, desde la propuesta creativa del usuario-destinatario al seleccionar unos textos y no otros:

“... recibo la información disponible en Internet...”;

“... bajo la información de Internet que me interesa...";

"... tengo una lista de páginas favoritas que leo con frecuencia";

"... estoy inscrita en una página donde te mandan monografías, aquí hay que escoger las que se pueden necesitar para las clases...";

"... selecciono alguna página que me interesa...";

“... bajo artículos para trabajos...”;

“... las herramientas tecnológicas ahora ofrecen la facilidad del proceso copiar-pegar...".

3. De acuerdo a la iniciativa en la construcción de textos: Los usuarios elaboran sus páginas con información procesada al margen del sistema (preconceptos), con datos recogidos en la propia red y vínculos que establecen a manera de hipertextos. Se identifica en esta modalidad de comunicación un 
rol activo-creativo del usuario que lo transforma no sólo en receptor y decodificador de datos, sino en codificador de textos para otros:

“... creo mi propia página con cosas que conozco y con la información que obtengo de Internet, con vínculos y otras herramientas...";

“... para los cursos de informática educativa he diseñado mi propia página web con direcciones de interés en el tema de la página...”;

“... más que nada busco la información y la complemento con la información que busco en Internet ...".

De esta manera, la comunicación en Internet no es única sino múltiple. Los modos de comunicarse van desde la interactividad en línea hasta la decodificación activa y la construcción creativa de textos. Estas intervenciones del usuario implican, sobre todo, un protagonismo para completar el circuito comunicativo. Como nunca antes la comunicación está determinada por el sujeto destinatario, más que por el sistema o el soporte emisor.

\section{RELACIONES DE COMUNICACIÓN}

El desarrollo de las comunicaciones en red ha tenido un impacto en el proceso de acumulación, procesamiento y recuperación de la información, pero también ha significado, un cambio en la relación entre el usuario y el propio medio. El destinatario se transforma en usuario, lo que implica reestructurar su relación con los conjuntos textuales inmersos en el sistema tecnológico digital, tanto en el nivel de la relación material con el componente "duro" o el soporte de átomos, como en el "blando" o de bits, en la nomenclatura de Negroponte.

Los alumnos entrevistados reconocen que su relación con la tecnología ha cambiado cuando han sido capaces de manejar los códigos que les permiten no sólo crear sus propios textos (páginas web, presentaciones, software educativo), sino interactuar en línea con otros usuarios, incluidos sus profesores.

Si el análisis se remite a la variable tecnológica, como usuarios establecemos un vínculo directo con las redes de computación y, en general, con los sistemas de comunicación digital. En las relaciones comunicativas de esta naturaleza el sujeto se vincula con el sistema según las posibilidades que están previamente ordenadas y definidas, de tal manera que "se puede hablar de interacción comunicativa también en los casos de las relaciones de uno o más sujetos con un texto y de las relaciones de uno o más sujetos con una máquina" ${ }^{\text {"12 }}$. Esto es, al seleccionar un texto, existe un producto "nuevo" que da origen a una situación no

\footnotetext{
12 BetTetiNI, G. y COLOMBO, F., "Las nuevas tecnologías de la comunicación", Barcelona, Paidós, 1995, pp. 16-17.
} 
totalmente precodificada, desde donde emerge la "comunicabilidad" o nexo entre el usuario y el objeto tecnológico.

La potencialidad de Internet, constituiría, entonces, una de las condiciones necesarias, pero no suficientes, para la realización del texto o del intercambio comunicativo, pues también forman parte del proceso el conocimiento previo sobre el funcionamiento del sistema computacional, es decir, las "competencias tecnológicas" o capacidad para manejar un lenguaje especializado.

Los alumnos entrevistados señalaron como indispensable, y lo cual determinaría la "comunicabilidad" de la experiencia tecnológica, la competencia material para desenvolverse con propiedad en este lenguaje, que no sólo se aprende en el aula, sino que de manera determinarte a través de los pares y de forma autónoma (Tabla 1).

Tabla 1: Interacción de acuerdo al lugar de conocimiento de competencias tecnológicas

\begin{tabular}{|l|c|c|c|c|c|}
\hline & \multicolumn{4}{|c|}{ Lugar donde aprendió a utilizar el computador } \\
\hline $\begin{array}{l}\text { Nivel de } \\
\text { interacción }\end{array}$ & Básica & Liceo & Casa & Amigos & Universidad \\
\hline Interactiva & $22 \%$ & $36 \%$ & $23 \%$ & $10 \%$ & $9 \%$ \\
\hline Creativa & $21 \%$ & $41 \%$ & $14 \%$ & $7 \%$ & $17 \%$ \\
\hline Informacional & $24 \%$ & $40 \%$ & $16 \%$ & $7 \%$ & $13 \%$ \\
\hline
\end{tabular}

\section{NIVELES DE INTERACCIÓN}

El trabajo de investigación ha puesto énfasis en la noción de "interacción". Entendemos este concepto en su sentido más amplio, como son las secuencias de acciones entre varias personas (o textos) y donde existe una participaciónintervención. Sabemos que a través de Internet los usuarios construyen su propia red de interacciones, tanto en un sentido interpersonal (de naturaleza humana) como en la participación del usuario con el dispositivo material para decodificar los textos disponibles.

De acuerdo a los rangos de intervención del sujeto para la construcción del texto se identifican tres niveles de interacción:

- Mínima-informacional, centrada en la selección, decodificación y acopio de datos disponibles en Internet, a partir de los cuales el usuario construye un texto propio. 
- Mediana-creativa, consiste en la creación de nuevos referentes desde los datos seleccionados y decodificados en la red, que se organizan a partir de los conceptos previos e intereses del usuario, ya sea como página web o software educativo.

- Máxima-interactiva, se ha incluido la modalidad de comunicación sincrónica y asincrónica, considerando tanto el uso del correo electrónico como la participación en foros de discusión y en chat rooms.

Esta distinción nos ha permitido un análisis del proceso comunicativo que efectivamente materializan los estudiantes universitarios en el uso que hacen de Internet, y cuáles constituyen los elementos que determinarían tales niveles de interacción.

Respecto a las preferencias en el uso de internet la mayoría reconoce su uso predominante para buscar información, consulta a bases de datos, utilización de correo electrónico y "chateo», mientras que dos de cada diez estudiantes sólo utiliza Internet para buscar información (Tabla 2). El alto porcentaje de estudiantes que utiliza el medio de manera creativa responde al diseño curricular de las carreras pedagógicas, que incluye, entre las actividades a desarrollar por parte de los estudiantes, la creación de páginas web y/o el desarrollo de software educativo. Sin embargo, no sólo los alumnos (as) de niveles superiores reconocen esta actividad creativa, sino aquellos estudiantes de los primeros semestres que aún no tienen como requisito académico esta actividad.

\section{Tabla 2}

\begin{tabular}{|c|c|}
\hline Nivel de interacción & $\%$ \\
\hline Informacional & 20 \\
\hline Creativa & 36 \\
\hline Interactiva & 44 \\
\hline
\end{tabular}

En la evaluación de la frecuencia en el uso de sistemas de comunicación sincrónica desde los lugares donde se tiene acceso a Internet, el mayor porcentaje se presenta en los cibercafés y en los propios domicilios (Tabla 3), en una proporción menor en la Universidad, pese a que los estudiantes disponen de equipamiento para tal fin. Respecto a los rangos de edad, los estudiantes más jóvenes y los de semestres superiores son los más proclives a utilizar Internet como mecanismo de comunicación en línea, mientras que los estudiantes en el rango intermedio, que, en general, aprendieron el uso de la herramienta en la propia Universidad, se limitan a un uso informacional del recurso (Tabla 4). 
Tabla 3: Frecuencia en la comunicación sincrónica por lugar de acceso a Internet

\begin{tabular}{|l|c|c|c|c|c|}
\hline & \multicolumn{5}{|c|}{ Frecuencia en el acceso } \\
\hline $\begin{array}{l}\text { Lugar de } \\
\text { acceso }\end{array}$ & Siempre & Casi siempre & Algunas veces & Casi nunca & Nunca \\
\hline Universidad & $16 \%$ & $22 \%$ & $37 \%$ & $18 \%$ & $7 \%$ \\
\hline Casa & $20 \%$ & $20 \%$ & $31 \%$ & $20 \%$ & $9 \%$ \\
\hline Cibercafé & $30 \%$ & $40 \%$ & $20 \%$ & $10 \%$ & $0 \%$ \\
\hline Amigos & $13 \%$ & $13 \%$ & $0 \%$ & $63 \%$ & $11 \%$ \\
\hline
\end{tabular}

Tabla 4: Frecuencia en la comunicación sincrónica por rangos de edad

\begin{tabular}{|l|c|c|c|c|c|}
\hline & \multicolumn{5}{|c|}{ Frecuencia en el acceso } \\
\hline $\begin{array}{l}\text { Rangos de } \\
\text { edad }\end{array}$ & Siempre & Casi siempre & Algunas veces & Casi nunca & Nunca \\
\hline $18-20$ & $24 \%$ & $27 \%$ & $24 \%$ & $19 \%$ & $6 \%$ \\
\hline $21-23$ & $14 \%$ & $18 \%$ & $37 \%$ & $19 \%$ & $12 \%$ \\
\hline $24-26$ & $15 \%$ & $12 \%$ & $47 \%$ & $25 \%$ & $0 \%$ \\
\hline 27 y más & $26 \%$ & $37 \%$ & $37 \%$ & $0 \%$ & $0 \%$ \\
\hline
\end{tabular}

Al explorar la relación entre el uso de la comunicación interactiva y el año de ingreso a la educación superior (Tabla 5 y 6), los datos son más que preocupantes respecto a la eficiencia del currículo en la Universidad, pues los mayores porcentajes en la frecuencia de uso de la comunicación interactiva están en los estudiantes de ingreso reciente (2002 y 2003), mientras que aquellos de cursos superiores no utilizan con frecuencia o simplemente no utilizan los sistemas de comunicación interactiva. Es decir, el desarrollo de las competencias tecnológicas hacia el uso de estos dispositivos comunicacionales ha sido más determinante en la educación 
básica y media que la superior, aún cuando en el currículo de formación pedagógica actualmente se pone énfasis en la alfabetización mediática, en la perspectiva de complementar la educación presencial universitaria con sistemas de formación virtual.

Tabla 5: Frecuencia en el uso de comunicación interactiva de acuerdo al año de ingreso

\begin{tabular}{|c|c|c|c|c|c|}
\hline & \multicolumn{5}{|c|}{ Frecuencia en el uso } \\
\hline Ingreso & Siempre & Casi siempre & Algunas veces & Casi nunca & Nunca \\
\hline 1999 & $0.4 \%$ & $0.4 \%$ & $0.4 \%$ & $0.4 \%$ & - \\
\hline 2000 & $2.3 \%$ & $4.6 \%$ & $6.2 \%$ & $2.7 \%$ & $0.4 \%$ \\
\hline 2001 & $4.6 \%$ & $3.0 \%$ & $7.3 \%$ & $4.6 \%$ & $3.0 \%$ \\
\hline 2002 & $4.6 \%$ & $4.2 \%$ & $9.2 \%$ & $5.8 \%$ & $2.3 \%$ \\
\hline 2003 & $6.9 \%$ & $9.6 \%$ & $9.2 \%$ & $5.8 \%$ & $1.5 \%$ \\
\hline
\end{tabular}

Tabla 6: Modalidad de interacción de acuerdo al año de ingreso a la Universidad

\begin{tabular}{|l|c|c|c|c|c|c|}
\hline & \multicolumn{2}{|c|}{ Interactiva } & \multicolumn{2}{c|}{ Creativa } & \multicolumn{2}{c|}{ Informacional } \\
\hline Ingreso & $f$ & $\%$ & $f$ & $\%$ & $f$ & $\%$ \\
\hline 2000 & 13 & 31 & 19 & 45 & 10 & 24 \\
\hline 2001 & 22 & 37 & 23 & 39 & 14 & 24 \\
\hline 2002 & 35 & 52 & 24 & 35 & 9 & 13 \\
\hline 2003 & 43 & 50 & 26 & 30 & 17 & 20 \\
\hline
\end{tabular}

\section{A MODO DE CONCLUSIÓN}

Tal como lo anticipaba McLuhan hace casi cuarenta años cualquier modificación de los medios produce una cadena de consecuencias en todos los niveles de la cultura y la política, y, fundamentalmente, en nuestras pautas de percepción. Los "internautas" contemporáneos en definitiva hemos alterado nuestros sentidos y nuestros modos de aprehender, pero tal proceso no se presenta en forma automática ni homogénea, sino que los "modos" de comunicarse son variados, diversos y 
están determinados por factores tanto socioculturales como políticos, entendidos estos como las pautas para permitir la accesibilidad material o alfabetización tecnológica de los usuarios.

De esta manera, en la categorización propuesta de los niveles de interacción posibles a través de Internet, identificamos tres posibilidades: la informacional, que estaría centrada en la selección, decodificación y acopio de datos disponibles en Internet, a partir de los cuales el usuario construye un texto propio; la creativa, que consistiría en la creación de nuevos referentes desde los datos seleccionados y decodificados en la red, que se organizan a partir de los conceptos previos e intereses del usuario, ya sea como página web o software educativo, y el modo de interacción máxima, donde se incorpora la modalidad de comunicación sincrónica y asincrónica, mediante el uso del correo electrónico como la participación en foros de discusión y en chat rooms.

Hemos identificado algunos factores que están presentes y de algún modo determinarían en uno u otro nivel las competencias tecnológicas, no asociadas directamente al lapso de tiempo en que el individuo utiliza la herramienta, sino al acceso más temprano a los procesos de alfabetización digital. Es decir, en nuestra estructura estudiantil tenemos alumnos jóvenes con una alta competencia tecnológica y alumnos de niveles superiores (segundo y tercer año) con una competencia tecnológica básica.

Debemos destacar que no se trata de concebir el uso de Internet como medio para la interacción o no, sino del uso más o menos frecuente de éste, que implica una apropiación del sistema, y con ello una consecutiva modificación de los modos de comunicarse.

Otro elemento a enfatizar es el componente de esparcimiento que implica el uso de Internet entre los estudiantes universitarios, pues si bien se reconoce su acceso como un apoyo a su formación profesional, la interacción que establecen a través del chat y los foros de discusión es preferentemente como distracción.

A partir de los resultados de este trabajo exploratorio, es indudable que la investigación sobre los procesos comunicativos o cognitivos que se materializan a través de la relación de los usuarios con los nuevos medios es una tarea pendiente, fundamentalmente lo que ha ocurrido en los últimos años con la reorganización de nuestros sentidos a partir de la incorporación de Internet como medio de comunicación. Si la radiodifusión significó una revolución social y, fundamentalmente, sensorial por parte de las audiencias de los años veinte, sin duda que Internet nos ha modificado algo más que nuestros hábitos de aproximación a la información y a "los otros", para interferir abiertamente en la organización de los sentidos y las formas de apropiarnos sensorialmente del mundo que nos rodea. 


\section{REFERENCIAS:}

BETTETINI, G. y Colombo, F., Las nuevas Tecnologías de la Comunicación, Barcelona, Paidós, 1995.

DAHNKE, G. L., "Investigación y comunicación”, En Fernández-Collado, C. y Dahnke G. L. (comps.), La comunicación humana, ciencia social, México, Mc-Graw-Hill, 1996, pp. 385-452.

DE VECCHI, B., Nuevas formas de vida, nuevas estructuras de comunicación, Hyperpage, Recuperado el 20 de diciembre del 2000, de http://www.kweb.it/hyperpage/ biblio1.htm

GUTIÉRREZ, A., Alfabetización digital. Algo más que ratones y teclas, Barcelona, Gedisa, 2003.

KRAMER, S. Dialogicita telemática? Tesi sula comunicazione en Internet, Rivista Sociología della Comunicazione, Istituto de Scienza dello Spettacole e Sociología della Comunicazione, Recuperado el 30 de octubre de 1997, de http://uiurb.it/ISCCS/ dialogic.html

SAAD, E. Presença digital da universidade: em busca da excelência colectiva, Recuperado el 2 de mayo, 2005, Disponible en: http//njmt.incubadora.fapesp.br/portal/publi/ beth/jornaluspdez00

SANCHO, J., "Enfoques y funciones de las nuevas tecnologías para la información y la educación: lo que es no es lo que parece", En De Pablos, J. y Jiménez, J. Nuevas tecnologías, comunicación audiovisual y educación, Barcelona, Cedenc, 1998, pp. 71 102.

THOMPSON, J., Ideología y cultura moderna. Teoría crítica social en la era de la comunicación de masas, México, UAM-Xochimilco, 1993.

WILLIAMS, F. y otros., Research Methods and the New Media. New York, Free Press, 1998.

WOLTON, D., Internet, ¿y después?, Barcelona, Gedisa, 2000.

(Recibido el 28-02-06, publicado el 16-05-06) 